Trauma Berufskrankh 2006 • 8[Suppl 2]: S195-S197 DOI 10.1007/s10039-005-1021-y

Online publiziert: 25. Juni 2005

(c) Springer Medizin Verlag 2005
W. Herfen · Düsseldorf

\section{Datenaustausch mit Leistungserbringern in der gesetzlichen Unfallversicherung}

\section{Erste Erfahrungen einer Durchgangsarztpraxis}

$I_{\mathrm{n}}$ n meiner Praxis erfolgt der Datenaustausch mit Leistungserbringern in der gesetzlichen Unfallversicherung - BLB (Bundesverband der landwirtschaftlichen Berufsgenossenschaften e. V.), BUK (Bundesverband der Unfallkassen), HVBG (Hauptverband der gewerblichen Berufsgenossenschaften) - seit nunmehr etwa 1,5 Jahren elektronisch. Die wesentlichen Gründe für die Einführung von DALEUV waren v. a.:

1. Schnellere Übermittlung der Berichte an die $\mathrm{BG}$

- Versand am gleichen Tag

- Vorlage des Berichts beim Sachbearbeiter am nächsten Morgen

- Zügige Weiterbearbeitung möglich

2. Kosten- und Zeitersparnis für Arzt und BG

a) für den Arzt

Keine Formulare oder Vordrucke

Kein Einkuvertieren und

Frankieren

Kein Gang zum Briefkasten

b) für die BG

Kein Porto, stattdessen Ziffer 192

UV-GOÄ

Keine manuelle Bearbeitung der

Patientendaten

3. Fehlerfreie Erfassung der

Patientendaten

- Übernahme aus der Stammdatei

- Vollständige Erfassung der unfallrelevanten Daten (Fehlermeldungen)

\section{Einsatz in der Praxis}

\section{BG-Formulare}

Derzeit können folgende Formulare elektronisch versandt werden:

- Durchgangsarztbericht F10oo (früher D13)

- Nachschaubericht F2106 (früher D9a)

- Mitteilung über Änderung der besonderen Heilbehandlung F2222 (früher KD10)

- Zwischenbericht F210o (früher AV5)

- Rechnungsvordrucke F9990, F9992,

F9994 (früher R1-R3)

Im 2. Quartal folgen die Ergänzungsberichte in M1.

\section{Hardware}

Benötigt werden:

1. Praxisrechner mit Krankenkartenchiplesegerät und Internet-/Mailzugang Es ist darauf zu achten, dass der Provider (z. B. telemed) eine VCS-Datenschnittstelle anbietet (V: VDAP, C: „communication“, S: Standard; VDAP: Verband Deutscher Arztpraxis-Softwarehersteller e. V.)

2. Chipkartenlesegerät für Praxis-PIN [Security-Karte für den Zugang zum ZKM (zentrales Kommunikationsmodul)]
3. Chipkartenlesegerät für Arzt-PIN [Security-Karte für die elektronische Unterschrift]

In meiner Praxis wird ein PC (FujitsuSiemens Senic Pentium 4; 3,o GHz; 8o GB HD; 512 MB RAM; Netzwerk 100 MBit) mit Tastatur, 17"-Monitor, Chipkartenleser KVK, telemed-Zugang mit VCS, Chipkartenleser für Praxis-PIN und Kryptierung sowie Chipkartenleser für Arzt-PIN verwendet.

\section{Software}

Für DALE-UV sind erforderlich:

1. Ein Arztpraxisprogramm eines Softwareherstellers, der Mitglied des VDAP ist (z. B. CompuMED M1 für niedergelassene Ärzte oder CompuPLAN K1 für Ambulanzen) (- Abb. 1)

2. Software für Internet-/Mailzugang mit VCS-Schnittstelle

3. Software zur Einbindung der Security-Karten-Lesegeräte

\section{Kosten}

Es entstehen zusätzliche Kosten durch:

- Monatliche Grundgebühr für Online-Zugang (zurzeit 3,oo EUR für ISDN+7,0o EUR für VCS)

- Verbindungsentgelte (inklusive Telefonkosten) für 
Trauma Berufskrankh 2006

8[Suppl 2]: S195-S197

DOI 10.1007/s10039-005-1021-y

(C) Springer Medizin Verlag 2005

\section{W. Herfen}

\section{Datenaustausch mit Leistungs- erbringern in der gesetzlichen Unfallversicherung. Erste Erfahrungen einer Durchgangsarztpraxis}

\section{Zusammenfassung}

Nach anfänglichen Schwierigkeiten kann nunmehr in der Praxis eine positive Bilanz für das elektronische Versenden von Berichten an den Versicherungsträger gezogen werden (D-Arzt-Bericht, Nachschaubericht, Zwischenbericht und Mitteilung über Änderungen der besonderen Heilbehandlung). Insgesamt kann eine Zeit- und Kostenersparnis für Arzt und BG verzeichnet werden. Durch Nutzung des Intranets mit Praxis- und Arzt-PIN wird größtmögliche Datensicherheit erreicht. Fehlerquellen werden durch entsprechende Hinweise minimiert. Nach einer gewissen Eingewöhnung ist das System bedienerfreundlich.

\section{Schlüsselwörter}

DALE-UV · Elektronische

Datenübermittlung .

Eingewöhnungsphase $\cdot$ Vorteile

\section{Data exchange in statutary accident insurance. Preliminary experiences in an accident insurance practice}

\section{Abstract \\ After initial difficulties, a positive balance can be drawn for the electronic dispatching of reports to the insurer (D-report, looking-af- ter checking, interim report and report over changes in special welfare treatment). Ti- me and cost saving result for both the physi- cian and the insurance associations involved. By the use of intranets with doctor-office-pin and physician-pin, the greatest possible da- ta security is reached. Sources of error are min- imized by appropriate instructions. After a cer- tain learning curve, the system is user friendly. \\ Keywords \\ DALE-UV · Electronic data communication . Acclimatizing $\cdot$ Positive balance}

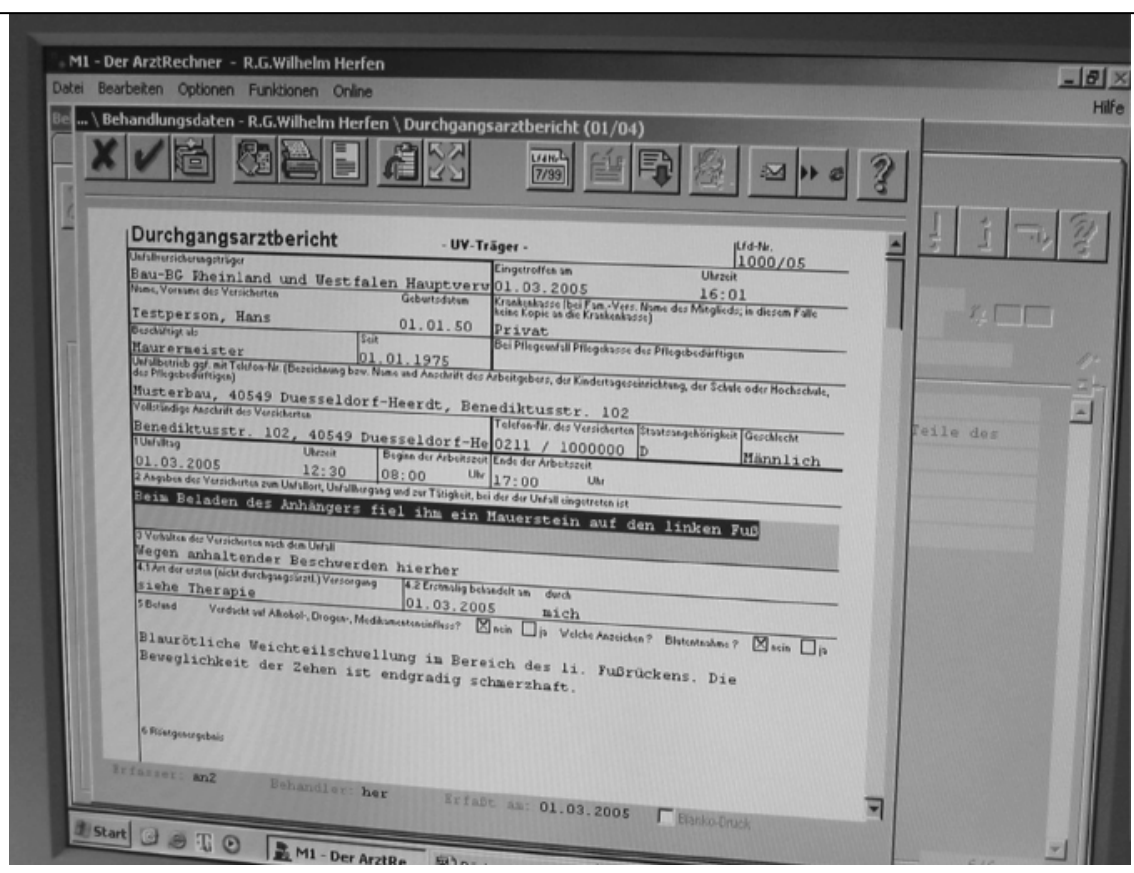

Abb. $1 \Delta$ Elektronischer D-Arzt-Bericht

a) Internet (z. B. T-Online)

b) Telemed-Intranet

- Freischaltung (einmalig, zurzeit 19,50 EUR)

- Kartenlesegerät für Praxis-PIN (einmalig, zurzeit 45,0o EUR)

- Kartenlesegerät für Arzt-PIN (einmalig, zurzeit 45,0o EUR)

Bei der Erstinstallation wird vom HVBG ein Zuschuss von 200,00 EUR gewährt.

Kostenlos sind:

- E-Mail-Postfach

- Electronic Banking

- Praxishomepage im Internet

Die monatlichen Kosten in meiner eigenen Praxis belaufen sich auf etwa 10,00 EUR bei etwa 120 versendeten Berichten und Rechnungen (inklusive Privatrechnungen).

\section{Sicherheit für Datenübertragung/ Schutz vor Eingriffen von außen}

Es gibt keine Zugangsmöglichkeit aus dem Internet ins Intranet. Durch PraxisPIN und Arzt-PIN besteht eine ausreichende Abschirmung der Daten.

Zurzeit ist bei zusätzlicher Nutzung des Internets über den Praxis-PC ein Eindringen von Viren möglich. Deshalb sollte derzeit ein Antivirusprogramm (z. B. McAfee) installiert sein
In Zukunft wird durch Vorschaltung eines Moduls nur noch der Zugang über das Intranet möglich sein. Dann sind nur noch Verbindungen zu arztpraxisrelevanten Stellen erlaubt (Abrechnungsstellen, KV, BG und sonstige Stellen des Gesundheitswesens). Dies wird mit Einführung der Gesundheitscard verpflichtend.

\section{Anfangsschwierigkeiten}

Vor etwa 1 1/2 Jahren begannen wir mit dem elektronischen Versenden der DArzt-Berichte. Die ersten Wochen waren von zahlreichen Pannen gekennzeichnet:

- Mangelnde Softwareeinbindung

- Versendung fehlerhafter Daten, die dann vom HVBG nicht weiter verarbeitet wurden

- Sperrung der Übersendung durch ein Antivirusprogramm

- Unkenntnis der Mitarbeiter der einzelnen Berufsgenossenschaften über das Verfahren

- Absturz des Programms

Mittlerweile sind diese Kinderkrankheiten beseitigt, und das System arbeitet zu $98 \%$ erfolgreich. Gelegentliche Fehler treten noch bei einigen Berufsgenossenschaften auf, die offensichtlich noch unvollständig elektronisch eingerichtet sind und bei denen die Mitarbeiter den D-Arzt-Bericht 
nicht erhalten. In diesen Fällen wird er per Fax übersandt.

Gelegentlich wird der Versand bei Überlastung des Internets blockiert, kann aber nach einer kurzen Wartezeit erfolgreich wiederholt werden.

\section{Ablauf der D-Arzt-Berichtaufnahme und Versendung}

1. Eingabe der Patientenstammdaten (Einlesen der Chipkarte oder Eingabe per Hand):

- Personendaten

- Arbeitgeber und BG

2. Anlage des BG-Behandlungsscheins mit den Unfalldaten

3. Aufruf des D-Arzt-Berichts in der Behandlungszeile

(Die Angaben aus Punkt 1 und 2 werden automatisch übernommen.)

4. Eingabe der Befunde und Diagnose mit ICD in den Bericht

5. Überprüfung der Daten vor dem Versand

6. Starten des Internetzugangs

7. Starten des ZKM (zentrales Kommunikationsmodul)

8. Eingabe der Praxis-PIN

9. Versenden des D-Arzt-Berichts mit Eingabe der Arzt-PIN

10. Überprüfen des korrekten Versands

\section{Tipp zur Rechnungserstellung}

Das Problem sind nicht die Rechnungserstellung und die Versendung, sondern die Begleichung durch die BG. Bei manchen Berufsgenossenschaften dauert sie Monate. Hinzu kommt die zeitaufwändige Überprüfung des Eingangs der Gelder und evtl. die Versendung von Mahnungen.

Wir rechnen nun über einen Abrechnungsservice (mediserv) ab. Die Rechnung wird erstellt und über das Intranet an die Abrechnungsstelle geschickt. Nach Abzug einer Bearbeitungsgebühr und der Portokosten kann ich den Betrag sofort auf das Praxiskonto überweisen lassen. Das spart Zeit und Geld. Die Vorteile dieses Vorgehens sind evident:

- Geringe Bearbeitungsgebühr

- 2,5\% Guthabenzinsen

- Sofortige Verfügbarkeit des Betrags

- Kein eigenes Mahnverfahren

\section{Resümee}

DALE-UV ist nach einer kurzen Eingewöhnungsphase ein einfaches, sicheres, Zeit und Kosten sparendes Übermittlungsverfahren.

\section{Korrespondierender Autor W. Herfen}

Benediktusstraße 78, 40549 Düsseldorf E-Mail:Wilhelm.Herfen@telemed.de

Interessenkonflikt: Der korrespondierende Autor versichert, dass keine Verbindungen mit einer Firma, deren Produkt in dem Artikel genannt ist, oder einer Firma, die ein Konkurrenzprodukt vertreibt, bestehen. 